\title{
Structure and Molecular Motion of Poly(ethylene oxide) Chains Tethered on Silica, Depended on Grafting Ratio by the Spin Labeled Method
}

\author{
Shigetaka ShImada, Atsushi MaruTA, and Katsuhiro Yamamoto
}

Nagoya Institute of Technology, Gokiso-cho, Showa-ku, Nagoya 466-8555, Japan

(Received July 12, 2000; Accepted September 4, 2000)

\begin{abstract}
Electron spin resonance (ESR) spectroscopy of spin-labeled poly(ethylene oxide) (SL-PEO) tethered on silica was studied to characterize the conformation and local dynamics of the chain end. The e.s.r spectra could be interpreted in terms of two components from two different kinds of spin labels attached to "train" and "tail" segments, which were strongly and weakly interacted with the silica surface. The structure and molecular motion of the chains are strongly dependent on the grafting ratio. The fractional amount of the "train" segment decreases remarkably with an increase in the grafting ratio. From the temperature dependence of the e.s.r. spectra, it was found that the molecular mobility of the "tail" segment was higher and that of the "train" segment was extremely lower than the PEO chains in the homopolymer bulk. These facts suggest that the "tail" segments are protruding from the silica surface and having a low segmental density, whereas the "train" segments are trapped near the silica surface. It can be also considered that the fractional amount of the "tail" segment increases abruptly with an increase in the grafting ratio after the tethered chains of a coiled structure form one monolayer.

KEY WORDS Tethered Chain / Silica / Molecular Motion / Molecular Density / ESR / Spin Label /
\end{abstract}

Many investigations have been published concerning adsorption of polymer on the silica surface. ${ }^{1-6} \mathrm{~A}$ few papers have been also presented about the adsorption behavior of polymer on the silica surface modified by different polymer chains. ${ }^{7,8}$ At present, it is very important to elucidate the structure and molecular motion of the tethered chains to confirm the adsorption behavior. In our previous paper, ${ }^{9,10}$ the spin label method was used to study the structure and molecular motion of the tethered poly(ethylene oxide)(PEO) chains on the silica having a low grafting ratio and the effect of polystyrene(PS) adsorption. It was concluded that the large PS molecules penetrated into the tethered $\mathrm{PEO}$ chain, which was strongly interacted with the silica surface, as "train" segments and the PEO chains protruded from the silica surface as "tail" segments. How does the structure of the tethered PEO chains change with an increase in grafting ratio? In this paper, we prepare several samples of silica-tethered PEO having different grafting ratios and clarify the structure and molecular motion of the PEO chains tethered on the silica surface as a function of grafting ratio. Tajouri et al. ${ }^{11,12}$ studied the organization of a layer of PEO chains tethered on silica by the nuclear magnetic resonance (NMR) relaxation method and concluded that the chains adopt a flat conformation on the surface at a low grafting ratio, whereas the chains adopt more extended conformation, perpendicular to the surface, at a high grafting ratio. The n.m.r. method characterizes the molecular mobility of the whole segments of the PEO chains.

On the other hands, the chain end site of the PEO molecules is spin-labeled and another chain end is bonded to the silica surface in the present study. The structure and molecular motion of the spin-labeled chain end should be affected by the interaction between the PEO segments and silica.

Then, it is expected that the distribution of the structure and molecular mobility can be elucidated.

\section{EXPERIMENTAL}

\section{Materials}

A Poly(ethylene oxide)PEO having $M_{\mathrm{w}}=2.0 \times 10^{3}$ was purchased from Katayama Science Co.. The PEO was purified by precipitating from two times from chloroform solution by addition of diethyl ether and dried under vacuum at room temperature for more than 1 day.

The non-porous silica gels were purchased from Nacalai Tesque Co. The specific surface area of silica is 376.5 $\mathrm{m}^{2} \mathrm{~g}^{-1}$, which are measured by the BET method (nitrogen adsorption method) using NOVA21000(Uasa Ionix Co.). The silica particles were purified by washing with acetone using a Soxlet apparatus and dried under vacuum at room temperature for more than 1 day.

\section{Preparation of PEO Chains Tethered on Silica Surface}

The grafting reaction was simply the direct esterification of a silica surface silanol group by a hydroxy end of the PEO. ${ }^{13}$ The reaction was performed in the polymer melt under at various temperatures in the range of 373 $\mathrm{K}$ and $453 \mathrm{~K}$ for $1 \mathrm{~h}$. The homopolymer was removed by the Soxlet extraction with acetone for 3 days. Finally, the grafted silica was dried under vacuum at room temperature for more than 1 day.

Determination of Grafting Ratio. The grafting ratio, GR (weight ratio of PEO to silica) of each sample was determined by pyrolysis of weight loss between $373 \mathrm{~K}$ and $573 \mathrm{~K}$. Thermogravimetry(TG) measurements were carried out by using a TG8101D calorimeter (Rigaku Denki Co.). TG scans were run from $298 \mathrm{~K}$ to $773 \mathrm{~K}$ at the heating rate of $5 \mathrm{~K} \mathrm{~min}^{-1}$. The GR ratio was calculated by the following equation, $\mathrm{GR}(\%)=(\mathrm{PEO}$ fraction $(\mathrm{g}) /$ Silica fraction $(g)) \times 100$

\section{Spin Labeling}

The ends of the tethered PEO chains and the PEO homopolymer were spin-labeled by the methods Törmälä 


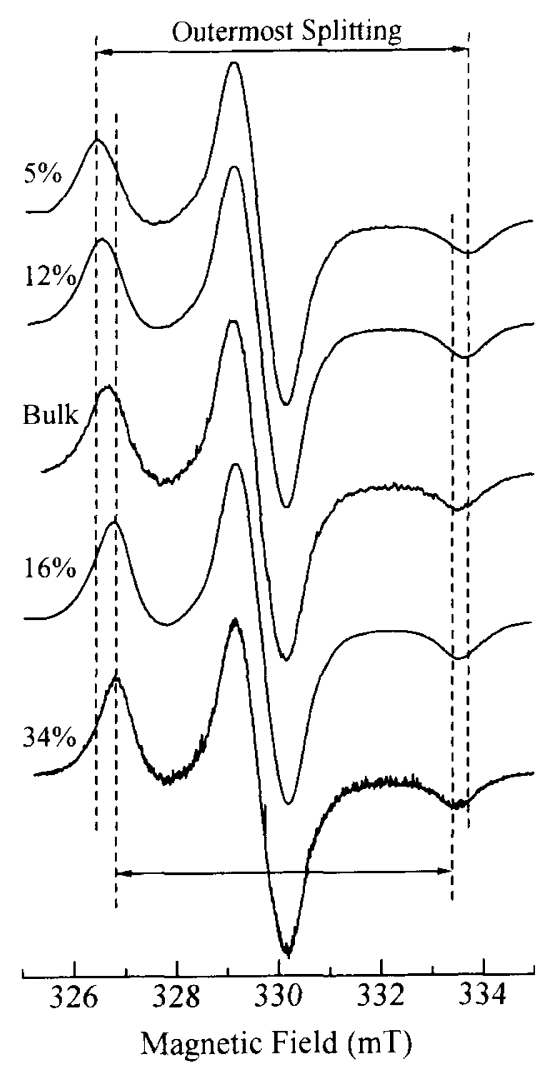

Figure 1. Variation of ESR spectrum of spin-labeld PEO tethered on silica with the grafting ratio. The measurements were carried out at $77 \mathrm{~K}$.

(a)

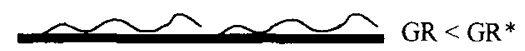

(b)

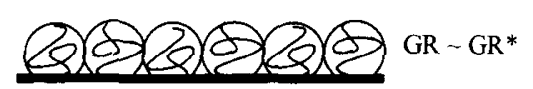

(c)

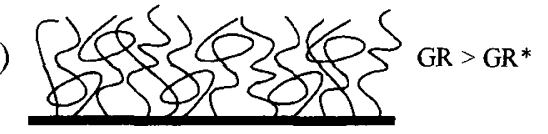

Figure 2. Schematic representation of structures of PEO segments tethered on the silica. Change of the conformation with the grafting ratio. The meaning of $\mathrm{GR}^{*}$ is mentioned in the text.

and Lindlberg. ${ }^{14}$ 3-Carbonyl-2,2,5,5-tetra-methyl-pyrroline-1-oxyl radical (Aldrich chemical Co.Ltd.) was esterified with the hydroxyl ends of the PEO. $0.2 \mathrm{~g}$ of the spin labeling reagent was used for $2.0 \mathrm{~g}$ of PEO. However, the degree of the spin labeling was extremely low and the concentration was lower than $10^{-4} \mathrm{~mol} \mathrm{~L}^{-1}$.

ESR Measurements. ESR spectra were observed in vacuo at a low microwave power level to avoid power saturation and with $100 \mathrm{kHz}$ field modulation using JEOL ME3XG and JEOL FE3XG spectrometers (Xband) at $77 \mathrm{~K}$ and above $113 \mathrm{~K}$, respectively, coupled to a microcomputer (NEC PC-9801). The signal of DPPH (1,1-diphenyl-2-picrylhydrazyl) was used as a g-value standard. The magnetic field was calibrated with the well-known splitting constants of $\mathrm{M}_{\mathrm{n}}{ }^{2+}$ in $\mathrm{MnO}$.

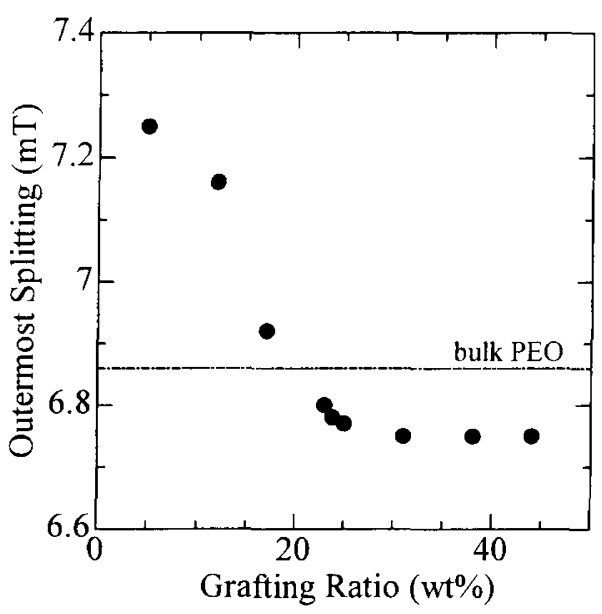

Figure 3. Variation of outermost splitting for SL-PEO in solid bulk and tethered on the silica with the grafting ratio. The ESR measurements were carried out at $77 \mathrm{~K}$.

\section{RESULTS AND DISCUSSION}

\section{Structure of PEO chains tethered on silica}

Figure 1 shows the dependence of ESR spectrum of spin-labeled PEO(SL-PEO) tethered on silica observed at $77 \mathrm{~K}$ on the grafting ratio (GR). The value of outermost splitting width for SL-PEO tethered on silica having GR of $6 \%$ is larger than those for SL-PEO in the bulk of PEO. The high value should be caused by the electric field of the silica surface as discussed in our previous papers. ${ }^{7}$ A spectrum with the high value of outermost splitting width for SL-PEO tethered on silica having GR of $5 \%$ in comparison with that in the PEO homopolymer bulk, reflects a strong $\mathrm{PEO}$-silica interaction. It can be considered that PEO segments connected with the SL$\mathrm{PEO}$ are strongly interacted with the silica as "train" segments as shown in Figure 2a.

On the other hand, the value of outermost splitting width for SL-PEO tethered on silica having GR of $25 \%$ is smaller than those in the PEO bulk. The experimental fact reflects a weak $\mathrm{PEO}$-silica interaction and a smaller effect of the electric field from PEO units than that in the homopolymer bulk. The small effect can be caused by the smaller molecular density around the chain ends for SL-PEO tethered on silica than that for SL-PEO in the homopolymer bulk. The segments connected with the SL-PEO behave like "tail" segments on the silica surface as shown in Figure 2c. The values of outermost splitting width at $77 \mathrm{~K}$ are plotted with GR in Figure 3 . It is found that the value, which reflects the structure of the tethered chain, has a strong dependence on GR. The outermost splitting decreases with an increase in GR for lower values than $20 \%$ and level off to $c a .6 .76 \mathrm{mT}$ lower than that for the homoplymer bulk. This result suggests the change of the fractional amount of "train" to "tail" segment with GR. The segments of the tethered PEO chains having a low GR interact strongly with the silica surface and take a "train" conformation. With an increase in GR, the tethered chain of a coiled structure cover the whole surface, form one monolayer and then, some of the segments begin to protrude from the surface 


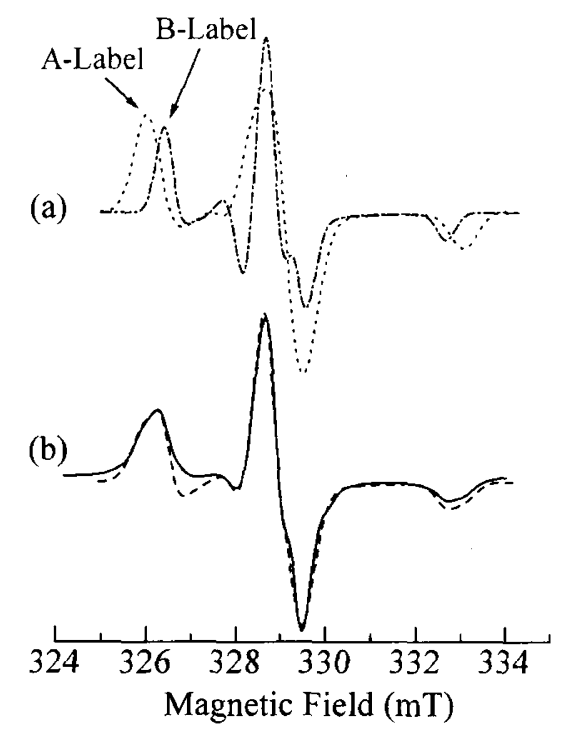

Figure 4. Comparison of observed (solid) and calculated (dashed) in the silica having the grafting ratio of $16 \%$ (b). The measurement was carried out at $233 \mathrm{~K}$. The calculated (dashed) in (b) is composed of two spectra in (a), which have larger (A-label) and smaller (B-label) extreme separation widths.

Table I. ESR parameters of spectra of SL-PEO tethered on the silica having the grafting ratio of $18 \%$ and in the homopolymer bulk, determined from the spectrum simulation mentioned in the text. The ESR measurements were carried out at $233 \mathrm{~K}$

\begin{tabular}{llll}
\hline \multirow{2}{*}{ Parameters } & \multicolumn{2}{c}{ L-PEO-g-Silica } & \\
\cline { 2 - 3 } & \multicolumn{1}{c}{$\mathrm{A}$} & $\mathrm{B}$ & L-PEO bulk \\
\hline$g_{\mathrm{x}}$ & 2.0095 & 2.0077 & 2.0099 \\
$g_{\mathrm{y}}$ & 2.0050 & 2.0058 & 2.0053 \\
$g_{\mathrm{z}}$ & 2.0018 & 2.0019 & 2.0018 \\
$g_{\text {iso }}$ & 2.0054 & 2.0051 & 2.0057 \\
$A_{\mathrm{x}}(\mathrm{mT})$ & 0.51 & 0.63 & 0.53 \\
$A_{\mathrm{y}}(\mathrm{mT})$ & 0.51 & 0.63 & 0.53 \\
$A_{\mathrm{z}}(\mathrm{mT})$ & 3.52 & 3.14 & 3.52 \\
$A_{\text {iso }}(\mathrm{mT})$ & 1.51 & 1.47 & 1.52 \\
Fraction $(\%)$ & 68 & 32 & 100 \\
\hline
\end{tabular}

and take a "tail" conformation with the contact of the tethered chains. It can be considered that many end parts of the tethered chain having GR of $16 \%$ take the "tail" conformation. To confirm the different conformation of "train" and "tail" segments, two components analysis of ESR spectra of SL-PEO was carried out. Figure 4 shows an example of ESR spectrum composed of two components. Both outermost peaks in the high and low magnetic field having shoulders can never been calculated by assuming only one component. In order to obtain the exact parameters and clarify the structures of the tethered PEO chains, spectral simulations for the ESR spectra observed at $77 \mathrm{~K}$ and $233 \mathrm{~K}$ are performed, firstly. The simulated spectrum(dashed line in Figure 4b) by assuming two components (dotted(A) and dotdashed(B) lines in Figure 4a) shows a good agreement with the observed spectrum at $233 \mathrm{~K}$ (solid line in Figure 4b). Here, the SL-PEO's, which give rise to larger and smaller extreme separation widths, are called to be Aand B-labels, respectively. This result indicates the classification of the spin-labels (A-labels and B-labels) hav-

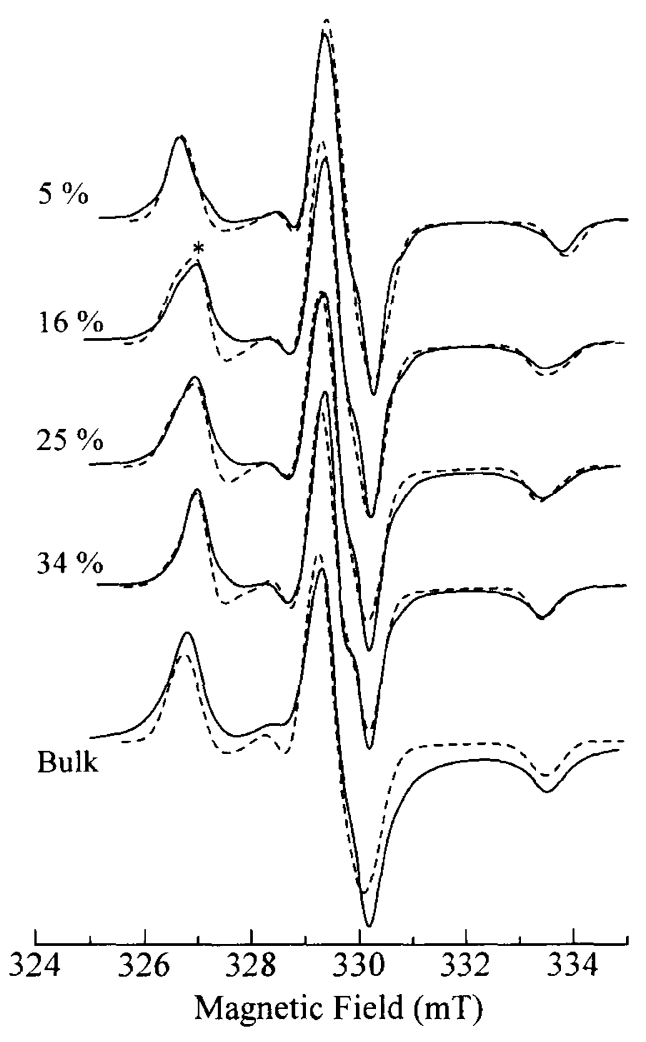

Figure 5. Variation of ESR spectrum of SL-PEO tethered on the silica with the grafting ratio. Solid and dashed lines indicate observed and calculated spectra, respectively. The measurements were carried out at $233 \mathrm{~K}$.

ing high and low values of hyperfine splitting (hfs) affected by different environments is a good approximation as discussed previously.

The ESR parameters of the A- and B-labels are determined from the spectral simulation and shown in Table I. The higher values of $A_{\mathrm{z}}$ and $A_{\text {iso }}$ for A-labels are caused by the strong electric field of the silica surfaces, for instance, the "train" segments connected with the A-labels have a strong PEO- silica interaction. The value of $A_{\text {iso }}$ for A-labels is larger than that of B-labels. On the other hand, the partial averaging of anisotropic hfs values for B-labels (low $A_{\mathrm{z}}$ and high $A_{\mathrm{x}}$ and $A_{\mathrm{y}}$ in comparison with those in the homopolymer $\mathrm{PEO}$ ) suggests that the molecular motion of the segments weakly interacted with the silica, has already occurred. It can be considered that A- and B-labels reflect "train" and "tail" segments, respectively as shown in Figure 2. Figure 5 shows the dependence of ESR spectrum of tethered SL-PEO on GR, observed at $233 \mathrm{~K}$. It should be noted that the shape of the outermost peaks change very remarkably with GR. For instance, the intensities of the innerpeaks in the peaks ${ }^{*}$ ) increase with an increase in GR. The simulated spectra show good agreements with the observed spectra. From the spectral simulation, it was found that the dependence of ESR spectra was caused by the variation of the fractional amount of B-labels with GR.

Figure 6 shows the dependencies of the fractional amounts of B-labels at $77 \mathrm{~K}$ and $233 \mathrm{~K}$ on GR. The fractional amounts of B-labels begin to increase steeply between $5 \%$ and $16 \%$ of GR and reach 0.45 and 0.62 at 77 $\mathrm{K}$ and $233 \mathrm{~K}$, respectively, for GR of $34 \%$. Consequently, 


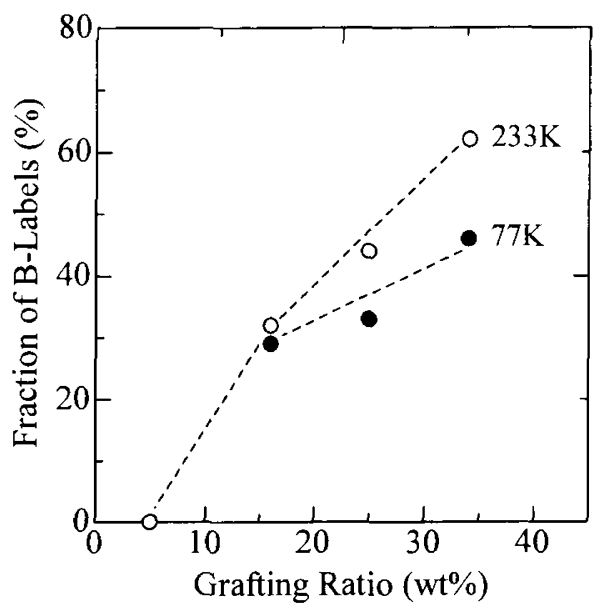

Figure 6. Fractional amount of B-labels of SL-PEO at $77 \mathrm{~K}($ and $233 \mathrm{~K}(\mathrm{O})$ as a function of grafting ratio from the spectrum simulation.

all segments of the tethered chains having lower GR than 5\% take the "train" conformation and then the conversion of the "train" to the "tail" conformation occurs with the increase in GR. These results suggest the different adsorption behaviors of the tethered PEO chains below and above the characteristic grafting ratio, GR*, at which the overlap of the PEO chains starts. The PEO chains are adsorbed on the silica surface and a flat conformation ("train") at low GR $<\mathrm{GR}^{*}$. On the other hand, at GR $>\mathrm{GR}^{*}$, the tethered PEO chains cover the whole surface of silica. For instance, an amount of PEO segments forms one monolayer on the surface. A tethered $\mathrm{PEO}$ chain occupied roughly a hemisphere with a radius comparable to the Flory radius, $R_{\mathrm{F}}$ for a coil. $R_{\mathrm{F}}=a N^{1 / 2}$, where $N$ is the number of repetitions and $a$ is the length of a segment. It can be obtained that a tethered PEO chain having $M_{\mathrm{w}}=2000$ occupies a part of the surface area of the silica given by $\pi R_{\mathrm{F}}^{2}=11.6 \mathrm{~nm}^{2}$. The schematic image of the structures of PEO chains which cover all silica surfaces is shown in Figure $2 \mathrm{~b}$. We calculate $0.108 \mathrm{~g}$ of $\mathrm{PEO}$ chains can form one monolayer on $1 \mathrm{~g}$ of silica, which has specific surface area, of $376.5 \mathrm{~m}^{2} \mathrm{~g}^{-1}$. Consequently, above the grafting ratio of $10.8 \%$, the PEO coils begin to overlap and protrude from the silica surface and a modification to the structure arises.

The calculated value of $\mathrm{GR}^{*}$ is between 5 and $16 \%$. Then, the fractional amount of B-labels attached to "tail" segments increases steeply.

In general, the adsorbed PEO segments have a distribution of conformation, which is a function of the degree of contact with the silica surface. At high GR, the PEO segments interrupt each other progressively from adopting the "train" conformation and the fractional amount of B-label, in other words, the probability of no contact of a PEO chain end with the silica surface, increase gradually with an increase in GR. High values at $233 \mathrm{~K}$ in comparison with those at $77 \mathrm{~K}$ in Figure 6 suggest that the thermal energy also change a part of "train" to "tail" conformation.

\section{Molecular Motion of PEO Chains Tethered on Silica}

In order to evaluate the molecular motion of the teth- (a)

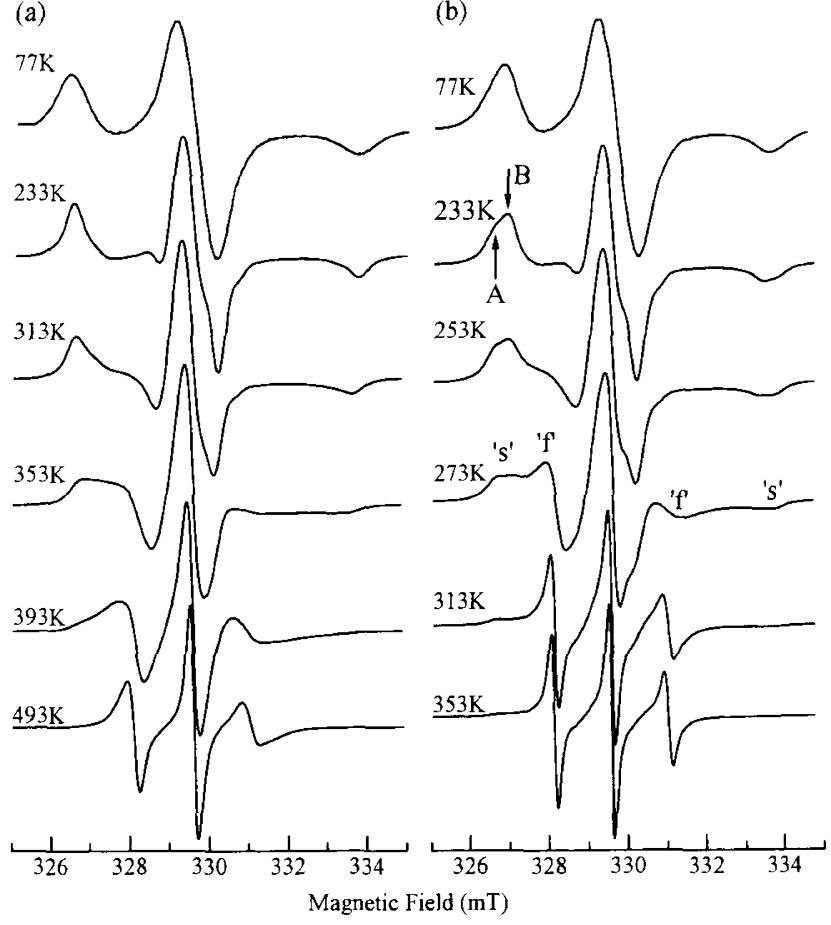

Figure 7. Temperature dependent ESR spectrum of SL-PEO tethered on the silica having the grafting ratio of $5 \%$ (a) and $16 \%$ (b). Slow (immobile) and fast (mobile) components are indicated as "s" and " $\mathrm{f}$ ", respectively. A- and B-labels are also indicated in the Figure.

ered PEO segments related to the PEO-silica interaction, ESR spectra were observed at various temperatures. Figure 7 shows temperature dependencies of ESR spectra of SL-PEO tethered on silica having GR's of 5\% (a) and 16\%(b). For the tethered PEO, multi-component spectra which are not remarkable for the homopolymer bulk are observed in the wide temperature range. The outermost splitting $\left(2 A_{z}{ }^{\prime}\right)$ for both A- and B-labels narrow with increasing motional averaging of the anisotropy of the hyperfine splitting due to the nitrogen nucleus. Spectral components ("s" and "f") of large and small $2 A_{\mathrm{z}}$ ' values reflect immobile and mobile labels, respectively. For instance, the A-labels convert from the immobile labels to mobile labels with conformational change of "train" segments to "tail" segments above temperatures where a micro Brownian type molecular motion occurs. On the other hand, the B-labels which take a "tail" segment and have a low molecular mobility at low temperatures also convert to the mobile labels when the micro Brownian type molecular motion starts. The change of A-labels to B-labels should be caused by the conversion of "train" segments to "tail" segments by local molecular motion, as discussed in the previous paper. ${ }^{7}$ Temperature where the conversion from immobile labels to mobile labels depends strongly on the magnitude of the interaction between the PEO chain end site and silica surface, regardless of the adsorbed structure of tethered segments. These facts suggest that the PEO segments have a very broad distribution of correlation time of molecular motion caused by the broad distribution of their adsorbed structure. The B-labels weakly interacted with silica surface change to mobile labels at lower tem- 


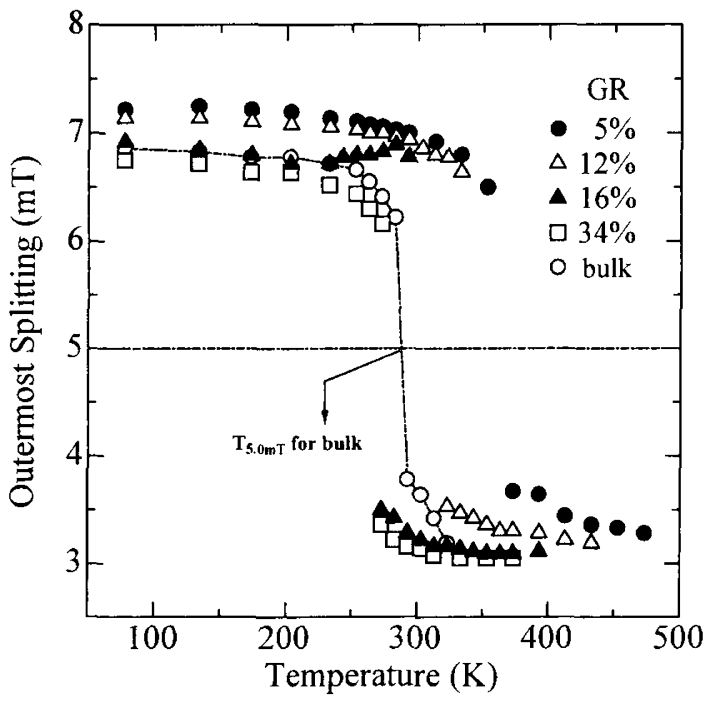

Figure 8. Variations of outermost splitting with temperature for SL-PEO in the homopolymer bulk $(O)$ and tethered on the silica having the grafting ratios: $5 \%(\mathbf{O}), 12 \%(\triangle), 16 \%(\mathbf{\Delta})$, and $34 \%$ ( $\square$ ).

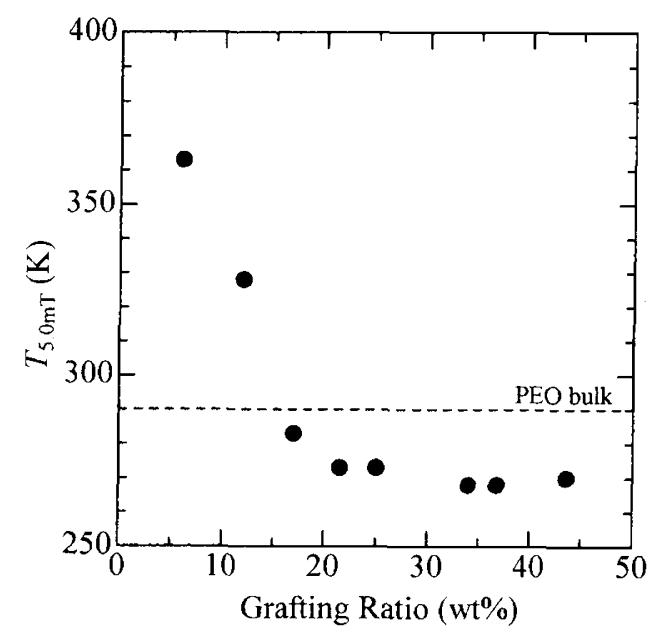

Figure 9. The dependence of $T_{5.0 \mathrm{mT}}$ on the grafting ratio. The value of $T_{5.0 \mathrm{mT}}$ for the homopolymer bulk is indicated as a dotted line.

peratures than the A-labels strongly interacted with that as "train" segments.

In general, the outermost splitting width of the main triplet spectrum due to hyper fine coupling caused by the nucleus narrows with an increase in mobility of the labels because of motional averaging of the anisotropic interaction between electron and nucleus. The complete averaging gives rise to the isotropic narrow spectrum..$^{15}$

It is found that the temperature dependence of the ESR spectra is a function of GR, related with the change in the structure and molecular motion of the PEO segments.

Transition of Immobile Labels to Mobile Labels. The outermost splitting for SL-PEO tethered on silica and in the homopolymer bulk are plotted against temperature in Figure 8. The value of the outermost splitting width as a function of temperatures is also a good measure of

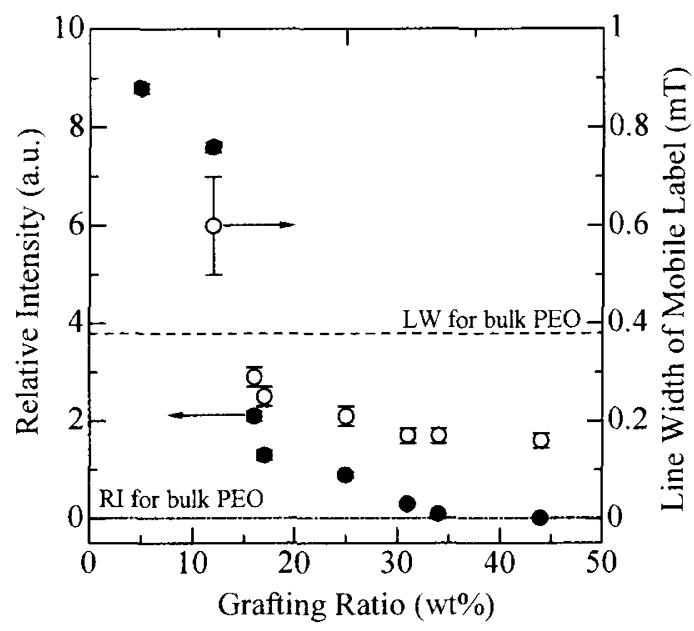

Figure 10. The dependencies of the relative intensity of immobile labels $($ ) and the line width of the spectrum for the mobile labels $(\bigcirc)$ on the grafting ratio. The values of the relative intensity and the line width for bulk PEO are indicated as dot-dashed and dashed lines, respectively.

the mobility of SL-PEO.

In the SL-PEO tethered on silica having GR of $5 \%$, the separation width drops between $340 \mathrm{~K}$ and $360 \mathrm{~K}$. On the other hand, the width drops between $250 \mathrm{~K}$ and 260 $\mathrm{K}$ for the samples having the higher GR values than $16 \%$. These transitions are caused by the conversion from the immobile labels of the tethered chains to the mobile labels which rotate more rapidly than the ESR frequency. The characteristic temperature, $T_{5.0 \mathrm{mT}}$, where the outermost splitting width decreases abruptly and reaches $5.0 \mathrm{mT}$ decreases with an increase in GR as shown in Figure 9. $T_{5.0 \mathrm{mT}}$ for the homopolymer bulk is between those for the silica-g-PEO having GR's of $12 \%$ and $17 \%$. These facts suggest that the magnitude of the interaction between silica and PEO segments of "train" conformation is much larger than the inter-segment interaction in the homopolymer bulk. On the other hand, the chain end site of "tail" conformation like "polymer brush", surprisingly, has extremely higher mobility than that in the homopolymer bulk which is due to a large free space around the end part.

Relative Amount of Immobile Labels. The relative intensity of the spectrum assigned to be immobile labels decreases with an increase in GR above $260 \mathrm{~K}$. Its dependence observed at $313 \mathrm{~K}$ is shown in Figure $10(\mathrm{O})$ as an example. The intensity of the immobile labels is proportional to be number of segment which interacts with the silica surface, regardless "of train" and "tail" segments. The very small intensity above $16 \%$ of GR indicates that almost all PEO chains protrude from the silica surface and behave as a polymer brush.

Mobility of mobile labels. The line width of spectrum assigned to be the mobile labels decreases with an increase in GR as shown in Figure 10( $\bigcirc)$. It is well known that the line width is an increasing function of correlation time of molecular motion. The large line width for the lower GR than $12 \%$ indicates that the mobile labels rotate more rapidly than ESR frequency, but still collide with the silica surface for the motion to be slowed down. 
On the other hand, the correlation decrease and the chain end of the mobile labels rotate rapidly in the free space with an increase in GR because of the separation of the end site from the silica surface.

The GR dependence of the molecular motion of the tethered chain is consistent with that of the structure as concluded in the previous section.

\section{CONCLUSION}

The structure and molecular motion of PEO chains tethered on silica surface are strongly dependent on the grafting ratio(GR).

1) When GR $<G^{*}$ at which the overlap of the PEO chains starts, all PEO chains take a flat conformation ("train" conformation) and interact strongly with silica surface and are suppressed from the molecular motion.

2) When GR $>G R^{*}$, the segments of the PEO chains released from the silica surface increase with an increase in GR take "tail" conformation like a "polymer brush" and have a high molecular mobility.

3) The chain ends of the tethered PEO chains in the structure like "polymer brush" have a low segmental density and rotate rapidly in the free space at high temperatures.

\section{REFERENCES}

1. A. Takahashi and M. Kawaguchi, Adv. Polym. Sci., 46, 1 (1982).

2. J. V. Alster and D. M. Ovenall, Polym. Commum., 32, 549, (1991).

3. A. K. Chakraborty and P. M. Adriani, Macromolecules, 25, 2470 (1992).

4. J. E. Gambogi and F. D. Bulum, Macromolecules, 25, 4526 (1992).

5. A. A. A. Ei-Hakim, A. M. Ramadan, and A. S. Badran, Polymer, 33, 4880 (1992).

6. H. D. Bijsterbosch, M. A. C. Stuart, and G. J. Fleer, Macromolecules, 31, 8981 (1998).

7. S. Shimada, K. Horiguchi, and K. Yamamoto. Colloid Polym. Sci., 276, 412 (1998).

8. S. J. Sofia, V. Premnath, and E. N. Merrill, Macromolecules, 31, 5059 (1998).

9 S. Shimada and T. Watanabe, Polymer, 39, 1703 (1998).

10. S. Shimada and T. Watanabe, Polymer, 39, 1711 (1998).

11. T. Tajouri and H. Bouchriha, Polymer, 37, 3185 (1996).

12. S. Azrizi, T. Tajouri, and H. Bouchriha, Polymer, 41, 5921 (2000).

13. H. Hommel, A. P. Legrand, H. B. Ouada, H. Bouchrihat, H. Baland, and E. Papirer, Polymer, 33, 181 (1992).

14. P. Törmälä and L. Lindlberg, Polymer, 14, 481 (1973).

15. "Spin Labeling, Theory and Application", L. J. Berliner, Ed., Academic Press, New York, N.Y., 1976. 